\title{
Nonsymmetric Variants of the Prekernel and the Prenucleolus*
}

\author{
Guni Orshan ${ }^{\dagger} \quad$ Peter Sudhölter ${ }^{\ddagger}$
}

\begin{abstract}
A solution on a class of TU games that satisfies the axioms of the pre-nucleolus or -kernel except the equal treatment property and is single valued for two-person games, is a nonsymmetric pre-nucleolus (NSPN) or -kernel (NSPK). In this paper we investigate the NSPKs and NSPNs and their relations to the positive prekernel and to the positive core. It turns out that any NSPK is a subsolution of the positive prekernel. Moreover, it is shown that an arbitrary NSPK, when applied to a TU game, intersects the set of preimputations whose dissatisfactions coincide with the dissatisfactions of an arbitrary element of any other NSPK applied to this game. This result also provides a new proof of sufficiency of the characterizing condition for NSPKs due to the first author in his PhD thesis published in 1994 as a discussion paper. Any NSPN belongs to "its" NSPK. Several classes of NSPNs are presented, all of them are subsolutions of the positive core. It is shown that any NSPN is a subsolution of the positive core provided that it satisfies the equal treatment property on an infinite universe of potential players. Moreover, we prove that, for any game that has a nonempty anticore, any NSPN selects its prenucleolus as its unique element.
\end{abstract}

Keywords: TU game $\cdot$ Solution concept $\cdot$ Kernel $\cdot$ Nucleolus $\cdot$ Core $\cdot$ Equal treatment

JEL Classification: $\mathrm{C} 71$

\section{Introduction}

The prenucleolus and the prekernel are widely acceptable solutions for cooperative transferable utility games. Introduced as auxiliary solutions of the prebargaining set, they became important solutions in their own rights, heavily supported by the fact that they can be justified by simple and intuitive axioms. Both are closely related, because they share many properties and because one, the prenucleolus, is a subsolution of the other. Two of these properties, anonymity (AN) and the equal treatment property (ETP), may be used, together with further axioms, to characterize these solutions (see Theorems 2.1 and $2.2)$.

This paper investigates the roles of AN and ETP in the aforementioned axiomatizations. Indeed, it may be desirable to apply a solution that has the properties of the pre-nucleolus or -kernel with the exception of AN or ETP. In order to mention one example of this kind, note that bankruptcy problems may be

*This research is supported by the Spanish Ministerio de Ciencia e Innovación under project ECO2009-11213, co-funded by the ERDF, and the second author was supported by the Edmund Landau Center for Research in Mathematical Analysis and Related Areas and by the Center for the Study of Rationality at the Hebrew University of Jerusalem.

${ }^{\dagger}$ Department of Agricultural Economics and Management, The Hebrew University, P.O. Box 12, Rehovot, 76100, Israel, and Department of Mathematics, The Open University of Israel, 1 University Road, P.O. Box 808, Raanana 43107, Israel; e-mail: orshan@agri.huji.ac.il

$\ddagger$ Department of Business and Economics, University of Southern Denmark, Campusvej 55, 5230 Odense M, Denmark; e-mail: psu@sam.sdu.dk 
modeled as cooperative TU games (see, e.g., Aumann and Maschler (1985)). However, in a bankruptcy problem, some of the creditors may be ranked so that ETP or AN may not be possible.

A solution that satisfies the axioms that characterize the pre-nucleolus except AN is called nonsymmetric prenucleolus (NSPN). Similarly, a nonsymmetric prekernel (NSPK) is a solution that assigns a single proposal to any 2-person game and satisfies the characterizing axioms of the prekernel except ETP.

The paper is organized as follows. In Section 2 the necessary notation and definitions, Peleg's (1986) axiomatization of the prekernel, and Sobolev's (1975) axiomatization of the prenucleolus and two variants (see Orshan (1993) and Orshan and Sudhölter (2003)) are presented. The set of NSPKs of a game is contained in the positive prekernel of the game. A preimputation belongs to the positive prekernel if it differs from some preimputation in the prekernel only inasmuch as it may assign different amounts to satisfied coalitions, that is, to coalitions that have negative excesses. The definition of the positive prekernel due to Sudhölter and Peleg (2000) is recalled in Section 3 in order to show that any NSPK is a subsolution of the positive prekernel. This proof uses Orshan's (1994) necessary and sufficient condition that characterizes the NSPKs, i.e., that guarantees nonemptiness (NE). Moreover, a new proof of sufficiency of the aforementioned condition is given. In fact, it is shown that, if $\sigma$ is an arbitrary fixed solution that satisfies the condition, then for any element $x$ of $\sigma$ applied to a TU game, any other solution that satisfies the condition, when applied to the same game, intersects the set of preimputations that treat all dissatisfied coalitions in the same way as $x$. Inserting the prekernal for $\sigma$, the foregoing statement shows NE of any solution that satisfies the condition.

The positive core of a game is the set of preimputations that assign the same dissatisfaction to the coalitions as the prenucleolus does. Hence, any NSPK intersects the positive core. Moreover, an NSPN is in "its" NSPK. In Section 4 several nontrivial classes of NSPNs are presented. Though it is not known whether any NSPN is contained in the positive core, it is shown that an NSPN coincides with the prenucleolus, when applied to a TU game that has a nonempty anticore (i.e., the dual game has a nonempty core, is balanced). Moreover, an NSPN is a subsolution of the positive core, provided that it treats an infinite set of potential players equally. The proof of this result (see Theorem 4.5) is based on a technical lemma (Lemma 4.6). Finally, Section 5 is devoted to the proof of this technical lemma.

It should be noted that the proofs of several new results use the characterization result of NSPKs, i.e., Theorem 3.4, whose proof was only published in a discussion paper of the first author (Orshan (1994)), a version of his $\mathrm{PhD}$ thesis. In the present paper we just recall some parts of the proof and give some sketches or hints about the remaining parts. We do not offer the complete proof, because it is not new (in fact more than 16 years old), quite technical, and contained in the aforementioned discussion paper. The authors should be happy to send an electronic copy of the discussion paper on demand to those readers who are interested in the details.

As mentioned above, in the beginning of the $1990^{\prime}$, the first author started investigating NSPKs. In fact, Michael Maschler supervised his $\mathrm{PhD}$ thesis and motivated the investigation of nonsymmetric prekernels. Almost at the same time, the second author tried to convince himself that AN is logically independent of the remaining axioms (see Sudhölter (1993)) in Sobolev's (1975) famous characterization of the prenucleolus. In this context, when asking whether the independence of AN was already known, Bezalel Peleg indicated that he could not exclude that a student of Maschler, namely the first author, knew this fact (which was true). Hence, Peleg initiated the joint research of both authors. Moreover, he was not only 
interested in this field of research as is documented by the existence of, e.g., a joint paper with the second author on the positive prekernel (2000), but also motivated the research; in fact he supervised the PhD research of the first author for one year, when Maschler was abroad.

\section{Notation, Solutions, and Properties}

Let $U,|U| \geq 4$, be the universe of players containing, without loss of generality, $1, \ldots, k \in U$ whenever $|U| \geq k$. A (cooperative TU) game is a pair $(N, v)$ such that $\emptyset \neq N \subseteq U$ is finite and $v: 2^{N} \rightarrow \mathbb{R}, v(\emptyset)=0$. For any game $(N, v)$ let

$$
X^{*}(N, v)=\left\{x \in \mathbb{R}^{N} \mid x(N) \leq v(N)\right\} \text { and } X(N, v)=\left\{x \in \mathbb{R}^{N} \mid x(N)=v(N)\right\}
$$

denote the set of feasible and Pareto optimal feasible payoffs (preimputations), respectively. We use $x(S)=\sum_{i \in S} x_{i}(x(\emptyset)=0)$ for every $S \in 2^{N}$ and every $x \in \mathbb{R}^{N}$ as a convention. Additionally, $x_{S}$ denotes the restriction of $x$ to $S$, i.e. $x_{S}=\left(x_{i}\right)_{i \in S}$, and we write $x=\left(x_{S}, x_{N \backslash S}\right)$. For $x \in \mathbb{R}^{N}, S \subseteq N$, and distinct players $k, \ell \in N$ let

$$
e(S, x, v)=v(S)-x(S) \text { and } s_{k \ell}(x, v)=\max _{S \subseteq N \backslash\{\ell\}: k \in S} e(S, x, v)
$$

denote the excess of $S$ and the maximum surplus ${ }^{1}$ of $k$ over $\ell$, respectively, at $x$ with respect to $(N, v)$. The prekernel (see Davis and Maschler (1965)) of $(N, v)$ is given by

$$
\mathcal{P K}(N, v)=\left\{x \in X(N, v) \mid s_{k \ell}(x, v)=s_{\ell k}(x, v) \text { for all } \mathrm{k} \in N, \ell \in N \backslash\{k\}\right\} .
$$

For $X \subseteq \mathbb{R}^{N}$ let $\mathcal{N}((N, v) ; X)$ denote the nucleolus of $(N, v)$ with respect to $X$, i.e., the set of members of $X$ that lexicographically minimize the nonincreasingly ordered vector of excesses of the coalitions (see Schmeidler (1969)). It is well-known that the nucleolus with respect to $X^{*}(N, v)$ is a singleton, the unique element of which is called the prenucleolus of $(N, v)$ and is denoted by $\nu(N, v)$.

In general, a solution $\sigma$ associates with each game $(N, v)$ a subset of $X^{*}(N, v)$. Let $\sigma$ be a solution. Then $\sigma$

(1) is covariant under strategic equivalence (COV) if, for all games $(N, v),(N, w)$ satisfying $w=\beta v+z$ for some $\beta>0$ and $z \in \mathbb{R}^{N}$, the equation $\sigma(N, w)=\beta \sigma(N, v)+z$ holds. (Here we use the convention that identifies $z \in \mathbb{R}^{N}$ with the additive coalitional function, again denoted by $z$, on the player set $N$ defined by $z(S)=\sum_{i \in S} z_{i}$ for all $S \in 2^{N}$. Also note that the games $v$ and $w$ are called strategically equivalent.);

(2) is nonempty (NE) if $\sigma(N, v) \neq \emptyset$ for every game $(N, v)$;

(3) is Pareto optimal $(\mathrm{PO})$ if $\sigma(N, v) \subseteq X(N, v)$ for every game $(N, v)$;

(4) is single-valued (SIVA) if $|\sigma(N, v)|=1$ for every game $(N, v)$;

(5) is anonymous (AN) if the following condition is satisfied for all games $(N, v)$. If $\pi: N \rightarrow U$ is an injection, then $\sigma(\pi(N), \pi v)=\pi(\sigma(N, v))$, where $\pi v(\pi(S))=v(S)$ for all $S \subseteq N$ and, for any $x \in \mathbb{R}^{N}, y=\pi(x) \in \mathbb{R}^{\pi(N)}$ is given by $y_{\pi(i)}=x_{i}$ for all $i \in N$ (in this case the games $(N, v)$ and $(\pi(N), \pi v)$ are isomorphic);

\footnotetext{
${ }^{1}$ Sometimes, if $(N, v)$ is fixed, we omit $v$ and simply write $e(S, x)$ and $s_{k \ell}(x)$.
} 
(6) satisfies the equal treatment property (ETP) if for every game $(N, v)$, for every $x \in \sigma(N, v), x_{k}=x_{\ell}$ for all substitutes $k, \ell \in N$ ( $k$ and $\ell$ are substitutes if $v(S \cup\{k\})=v(S \cup\{\ell\})$ for all $S \subseteq N \backslash\{k, \ell\})$;

(7) is reasonable (REAS) if, for every game $(N, v)$, for every $x \in \sigma(N, v)$, and for every $i \in N$,

$$
\min _{S \subseteq N \backslash\{i\}}(v(S \cup\{i\})-v(S)) \leq x_{i} \leq \max _{S \subseteq N \backslash\{i\}}(v(S \cup\{i\})-v(S))
$$

(8) satisfies the reduced game property (RGP) if for any game $(N, v)$, for every $\emptyset \neq S \subseteq N$, and any $x \in \sigma(N, v), x_{S} \in \sigma\left(S, v^{S, x}\right)$ (the reduced game $\left(S, v^{S, x}\right)$ is defined by $v^{S, x}(\emptyset)=0, v^{S, x}(S)=$ $v(N)-x(N \backslash S)$, and $v^{S, x}(T)=\max _{Q \subseteq N \backslash S}(v(T \cup Q)-x(Q))$ for $\left.\emptyset \neq T \varsubsetneqq S\right)$;

(9) satisfies the converse reduced game property (CRGP) if for every game $(N, v)$ with $|N| \geq 2$ the following condition is satisfied for every $x \in X(N, v)$ : If, for every $S \subseteq N$ with $|S|=2, x_{S} \in$ $\sigma\left(S, v^{S, x}\right)$, then $x \in \sigma(N, v)$;

(10) satisfies the reconfirmation property (RCP) if, for any game $(N, v)$, for every $\emptyset \neq S \subseteq N$, for any $x \in \sigma(N, v)$ and $y \in \sigma\left(S, v^{S, x}\right),\left(y, x_{N \backslash S}\right) \in \sigma(N, v)$.

For interpretations and discussions, in particular of the variants (8), (9), and (10) of the reduced game property, see Peleg (1986) and Hwang and Sudhölter (2001).

We now recall the classical characterizations of the foregoing solutions.

Theorem 2.1 (Sobolev (1975)) If $|U|=\infty$, then the prenucleolus is the unique solution that satisfies ${ }^{2}$ SIVA, COV, AN, and RGP.

Theorem 2.2 (Peleg (1986)) The prekernel is the unique solution that satisfies NE, PO, COV, ETP, RGP, and CRGP.

Of course, for a single valued solution $\sigma$ the axioms RGP and RCP are equivalent. So, we may replace RGP by RCP in Theorem 2.1. Surprisingly, if RCP is used together with ETP instead of AN, then it is possible to replace SIVA by NE so that we have the following result.

Theorem 2.3 (Orshan and Sudhölter (2003)) If $|U|=\infty$, then the prenucleolus is the unique solution that satisfies NE, COV, ETP, and RCP.

Remark 2.4 By means of an example, Peleg and Sudhölter (2003, Remark 6.3.3) show that both variants of Theorem 2.1 and Theorem 2.3 are no longer valid, if $4 \leq|U|<\infty$.

In view of the foregoing remark, a solution $\sigma$ is called a nonsymmetric prenucleolus (NSPN) if it satisfies SIVA, COV, and RGP, and if $|U|=\infty$.

In order to define nonsymmetric prekernels we do not simply delete ETP in Theorem 2.2, because there are many "pathological" examples (e.g., the solution $X(\cdot, \cdot)$ assigning the set of all preimputations to a game) that satisfy the remaining axioms. One basic property of the prekernel is kept. Indeed, the

\footnotetext{
${ }^{2}$ Orshan (1993) shows that AN may be replaced by ETP.
} 
prekernel of any two-person game coincides with its prenucleolus, so the prekernel of any two-person game is single valued. A solution $\sigma$ is 2-SIVA if $|\sigma(N, v)|=1$ for any game $(N, v)$ with $|N|=2$. We say that $\sigma$ is a nonsymmetric prekernel if $\sigma$ satisfies 2-SIVA, NE, PO, COV, RGP, and CRGP (see Orshan (1994)).

Remark 2.5 (1) A solution that satisfies PO, RGP, and CRGP, is uniquely determined by the 2person games in the following sense. If $\sigma$ is a solution that satisfies PO and RGP, then there exists a unique solution $\widetilde{\sigma}$ that satisfies PO, RGP, and CRGP, and coincides with $\sigma$ for any 2-person game. So CRGP may be replaced by "maximality" (see Remark 3.12 of Orshan (1994) or Remark 3.7 of Hwang and Sudhölter (2001)).

(2) As SIVA, COV, and RGP imply PO (see Sobolev (1975)), we conclude that every NSPN $\sigma$ is a subsolution of a unique NSPK $\widetilde{\sigma}$ defined by $\widetilde{\sigma}(N, v)=\sigma(N, v)$ for any game $(N, v)$ with $|N| \leq 2$.

\section{Nonsymmetric Prekernels and the Positive Prekernel}

In order to recall Orshan's (1994) complete characterization of NSPKs let $\sigma$ be a solution that satisfies 2-SIVA, PO, COV, RGP, and CRGP. In view of Remark 2.5 and of COV, $\sigma$ is determined as soon as it is defined for all 0-1 and 0-(-1) normalized 2-person games. (Indeed, up to strategic equivalence a 2-person game $(\{k, \ell\}, v)$ satisfies $v(\{k\})=v(\{\ell\})=0$ and $v(\{k, \ell\}) \in\{1,-1,0\}$.) However, if $v(\{k, \ell\})=0$, then, by 2-SIVA and COV, $\sigma(\{k, \ell\}, t v)=t \sigma(\{k, \ell\}, v)$ for $t>0$, so $\sigma(\{k, \ell\}, v)=\{0\}$ by NE. For $k, \ell \in U$, $k \neq \ell$, let $a_{k \ell}^{\sigma}=a_{k \ell}$ denote the $k$-coordinate of the unique element of $\sigma$ applied to the 0-1 normalized game on $\{k, \ell\}$ and let $b_{k \ell}^{\sigma}=b_{k \ell}$ be the $k$-coordinate of the $0-(-1)$ normalized game on $\{k, \ell\}$. By PO,

$$
\begin{aligned}
a_{k \ell}+a_{\ell k} & =1 \\
b_{k \ell}+b_{\ell k} & =-1 .
\end{aligned}
$$

Let $(N, v)$ be a game, let $x \in \sigma(N, v)$, let $k, \ell \in N, k \neq \ell$, and let $S=\{k, \ell\}$. If $s_{k \ell}(x)+s_{\ell k}(x)<0$, then the reduced game $\left(S, v^{S, x}\right)$ is strategically equivalent to the 0-1 normalized game on $S$ so that ${ }^{3}$ $a_{k \ell} s_{\ell k}(x)=a_{\ell k} s_{k \ell}(x)$. If $s_{k \ell}(x)+s_{\ell k}(x)>0$, then $\left(S, v^{S, x}\right)$ is strategically equivalent to the $0-(-1)$ normalized game so that $b_{k \ell} s_{\ell k}(x)=b_{\ell k} s_{k \ell}(x)$. Finally, if $s_{k \ell}(x)+s_{\ell k}(x)=0$, then $\left(S, v^{S, x}\right)$ is strategically equivalent to the "flat" ( $0-0$ normalized) game so that $0=s_{\ell k}(x)=s_{k \ell}(x)$. Hence, by RGP and CRGP,

$$
\sigma(N, v)=\left\{\begin{array}{l|l}
x \in X(N, v) & \begin{array}{l}
a_{k \ell} s_{\ell k}(x)=a_{\ell k} s_{k \ell}(x) \text { if } s_{k \ell}(x)+s_{\ell k}(x)<0 \\
b_{k \ell} s_{\ell k}(x)=b_{\ell k} s_{k \ell}(x) \text { if } s_{k \ell}(x)+s_{\ell k}(x) \geq 0
\end{array}
\end{array} \text { for all } k, \ell \in N, k \neq \ell\right\} .
$$

The converse is also valid: If for all distinct $k, \ell \in U, a_{k \ell}, b_{k \ell} \in \mathbb{R}$ satisfy (3.1)and (3.2) and if $\sigma$ is defined by (3.3), then $\sigma$ satisfies 2-SIVA, PO, COV, RGP, and CRGP.

An NSPK satisfies the foregoing 5 axioms and NE. In order to describe the impact of NE on the $a_{k \ell}$ and $b_{k \ell}$, we shall now briefly review Section 6 of Orshan (1994) and start with the following lemma.

Lemma 3.1 Let $\sigma$ be an NSPK and let any $k, \ell \in U$ with $k \neq \ell$. Then $a_{k \ell}^{\sigma} \geq 0$ and $b_{k \ell}^{\sigma}=-\frac{1}{2}$.

\footnotetext{
${ }^{3}$ Note that $s_{i j}(y):=s_{i j}(y, v)=s_{i j}\left(y_{T}, v^{T, y}\right)$ for $i, j \in T, i \neq j, \emptyset \neq T \subseteq N$, and $y \in \mathbb{R}^{N}$.
} 
Note that the $b_{k \ell}$ have no longer to be specified and (3.3) simplifies to

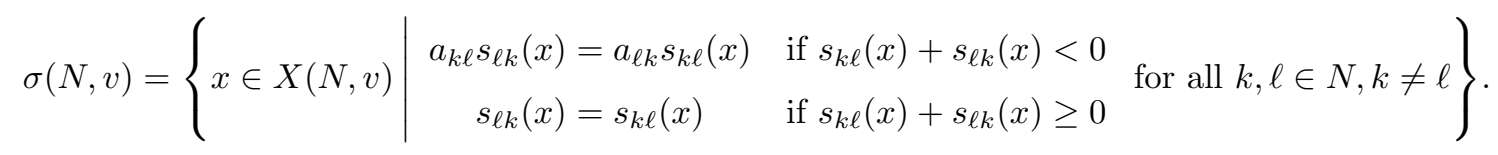

We do not present the proof of Lemma 3.1 that is technical and requires to consider several 3-person games. Instead we show that Lemma 3.1 implies that any NSPK is a subsolution of the positive prekernel whose definition is now recalled. Let $t_{+}=\max \{t, 0\}$ denote the positive part of a real number $t$. Let $(N, v)$ be a game and $x \in \mathbb{R}^{N}$. Note that the excess $e(S, x, v)$, if positive, is interpreted as dissatisfaction of $S$ and may be used in a bargaining process by players of $S$ to object against $x$ (see, e.g., Davis and Maschler (1967)). These considerations suggest to define the positive prekernel of a game $(N, v)$ to be the set

$$
\mathcal{P} \mathcal{K}_{+}(N, v)=\left\{x \in X(N, v) \mid s_{k \ell}(x)_{+}=s_{\ell k}(x)_{+} \text {for all } k, \ell \in N, k \neq \ell\right\} .
$$

For an analysis of the positive prekernel see Sudhölter and Peleg (2000), who present also the following axiomatization.

Theorem 3.2 The positive prekernel is the unique solution that satisfies NE, AN, REAS, RGP, CRGP, and is maximal with the foregoing properties.

Corollary 3.3 Any NSPK is a subsolution of the positive prekernel.

Proof: Let $\sigma$ be an NSPK, let $(N, v)$ be a game, let $x \in \sigma(N, v)$, and let $k, \ell \in N, k \neq \ell$. If $s_{k \ell}(x)+$ $s_{\ell k}(x)<0$, then (3.3) implies $s_{k \ell}(x), s_{\ell k}(x) \leq 0$, because $a_{k \ell}, a_{\ell k} \leq 0$ by Lemma 3.1. If $s_{k \ell}(x)+s_{\ell k}(x) \geq 0$, then (3.3) implies $s_{k \ell}(x)=s_{\ell k}(x)$, because $b_{k \ell}=b_{\ell k} \neq 0$ by Lemma 3.1. Hence, $x \in \mathcal{P} \mathcal{K}_{+}(N, v)$. q.e.d.

For any $k, \ell \in U, k \neq \ell$, let $a_{k \ell} \in \mathbb{R}$ and let $\boldsymbol{a}=\left(a_{k \ell}\right)_{k, \ell \in U, k \neq \ell}$. Then the mapping $\boldsymbol{a}$ is said to generate legal chains if for all distinct players $j, k, \ell \in U$ the following conditions are fulfilled:

$$
\begin{aligned}
a_{k \ell} & \geq 0 ; \\
a_{k \ell}+a_{\ell k} & =1 ; \\
a_{j k}=1=a_{k \ell} & \Rightarrow a_{j \ell}=1 ; \\
a_{j k}=\frac{1}{2}=a_{k \ell} & \Rightarrow a_{j \ell}=\frac{1}{2} ; \\
a_{j k} \notin\left\{0, \frac{1}{2}, 1\right\} & \Rightarrow a_{j \ell} \in\{0,1\} .
\end{aligned}
$$

Now we are ready for presenting the characterization.

Theorem 3.4 (Orshan (1994)) The solution $\sigma$ is an NSPK if and only if there exists a mapping $\boldsymbol{a}$ $=\left(a_{k \ell}\right)_{k, \ell \in U, k \neq \ell}$ that generates legal chains such that, for any game $(N, v), \sigma(N, v)$ is given by 3.4.

It should be noted that the proof of the remaining part of the "only if" direction. i.e., the verification of $(3.7)-(3.9)$, is also quite technical and not presented in this paper. Regarding the "if" direction we shall show a stronger result that is interesting in its own right. The following notation is needed. 
Let $\boldsymbol{a}=\left(a_{k \ell}\right)_{k, \ell \in U, k \neq \ell}$ generate legal chains, let $(N, v)$ be a game and let $k, \ell \in N, k \neq \ell$. Define, for $x \in X(N, v), f_{k \ell}(x)=f_{k, \ell}^{a}(x, v)$ by

$$
f_{k \ell}(x)= \begin{cases}\min \left\{s_{k \ell}(x)-s_{\ell k}(x), s_{k \ell}(x)-\frac{a_{k \ell}}{a_{\ell k}} s_{\ell k}(x)\right\} & \text {, if } a_{k \ell} \leq a_{\ell k}, \\ \max \left\{s_{k \ell}(x)-s_{\ell k}(x), \frac{a_{\ell k}}{a_{k \ell}} s_{k \ell}(x)-s_{\ell k}(x)\right\} & \text { if } a_{k \ell} \geq a_{\ell k} .\end{cases}
$$

The following lemma, due to Orshan (1994), is useful.

Lemma 3.5 Let $\boldsymbol{a}=\left(a_{k \ell}\right)_{k, \ell \in U, k \neq \ell}$ generate legal chains, let $(N, v)$ be a game, let $\sigma$ be defined by (3.4), and let $f$.. be defined by (3.10).

(1) For $k, \ell \in N, k \neq \ell$, the mapping $f_{k \ell}: X(N, v) \rightarrow \mathbb{R}$ is continuous.

(2) For any distinct $k, \ell \in N, f_{k \ell}=-f_{\ell k}$.

(3) $\sigma(N, v)=\left\{x \in X(N, v) \mid f_{k \ell}(x)=0\right.$ for all distinct $\left.k, \ell \in N\right\}$.

(4) For any $x \in X(N, v)$ the relation $\succeq_{x}$ on $N$ defined by $k \succeq_{x} \ell$ if $f_{k \ell}(x) \geq f_{\ell k}(x)$ for all $k, \ell \in N$, $k \neq \ell$, is a partial order relation, i.e., reflexive, antisymmetric, and transitive.

Proof of (1) - (3): Any excess function $e(S, \cdot): X(N, v) \rightarrow \mathbb{R}$ is continuous for any $S \subseteq N$ so that, as a maximum of such functions, $s_{k \ell}$ is also continuous and the continuity of $f_{k \ell}$ follows. Statements $(2)$ and (3) are straightforward consequences of (3.4) and (3.10), respectively.

q.e.d.

We just present a brief sketch of the proof of (4) of Lemma 3.5: Note that $\succeq_{x}$ is clearly reflexive and antisymmetric. Let $j, k, \ell \in U$ such that $j \succ_{x} k \succ_{x} \ell$, i.e., $f_{j k}(x)>f_{k j}(x)$ and $f_{k \ell}(x)>f_{\ell k}(x)$. The proof that $f_{j \ell}(x)>f_{\ell j}(x)$ is technical, requires to distinguish cases (see (3.5) - (3.9)), and uses, e.g., the transitivity of the "outweigh relation" ( $k$ outweighs $\ell$ if $s_{k \ell}(x)>s_{\ell k}(x)$, see Lemma 5.1 of Davis and Maschler (1965)).

Orshan (1994) used the foregoing lemma to show with the help of the KKM lemma that $\sigma(N, v)$ defined by (3.4) is nonempty, that is, the sufficiency ("if") part of Theorem 3.4. We shall now use an equivalent criterion, namely Brouwer's fixed point theorem, to prove a stronger result. To this end, let $x \in X(N, v)$ and denote

$$
Z=Z(N, v, x)=\left\{z \in X(N, v) \mid e(S, z)_{+}=e(S, x)_{+} \text {for all } S \subseteq N\right\} .
$$

So $Z$ is the set of all preimputations such that a coalition keeps its dissatisfaction at $x$ if it has some and all coalitions satisfied at $x$ remain satisfied.

Theorem 3.6 Let $\sigma$ and $\sigma^{\prime}$ be NSPKs, let $(N, v)$ be a game, and let $x \in \sigma^{\prime}(N, v)$. Then

$$
\sigma(N, v) \cap Z(N, v, x) \neq \emptyset \text {. }
$$

The foregoing theorem applied to $\sigma^{\prime}=\mathcal{P K}$ yields non-emptiness of any NSPK $\sigma$.

Proof: Let $n=|N|$ and $Z=Z(N, v, x)$. Then $Z$ is nonempty, compact, and convex. Define, for any $z \in Z, g(z)=y \in \mathbb{R}^{N}$ by

$$
y_{k}=z_{k}+\frac{1}{n^{2}} \sum_{\ell \in N \backslash\{k\}} f_{k \ell}(z) \text { for all } k \in N,
$$


where $f_{k \ell}$ is defined by (3.10). By (2) of Lemma 3.5, $y(N)=z(N)$ so that $y \in X(N, v)$. In order to show that $y \in Z$ it is remarked that, by Corollary 3.3 , for distinct $k, \ell \in N$,

$$
\begin{aligned}
& s_{k \ell}(z) \leq 0 \quad \Rightarrow \quad s_{k \ell}(z) \leq f_{k \ell}(z) ; \\
& s_{k \ell}(z)>0 \Rightarrow f_{k \ell}(z)=0 .
\end{aligned}
$$

Now, let $S \subseteq N, S \neq \emptyset$. Note that, again by (2) of Lemma 3.5,

$$
y(S)=z(S)+\frac{1}{n^{2}} \sum_{k \in S, \ell \in N \backslash S} f_{k \ell}(z) .
$$

Let $k \in S$ and $\ell \in N \backslash S$. We distinguish two cases. If $e(S, z)>0$, then $s_{k \ell}(z) \geq e(S, z)>0$ and, by (3.12), $y(S)=z(S)$ and $e(S, y)=e(S, x)$. If $e(S, z) \leq 0$, then, by (3.11) or (3.12) respectively, $f_{k \ell}(z) \geq e(S, z)$. As $|S| \cdot|N \backslash S|<n^{2}, e(S, y) \leq 0$. We conclude that $g(z) \in Z$.

Now the proof can be completed. By (1) of Lemma 3.5, $g: Z \rightarrow Z$ is continuous and, by Brouwer's fixed point theorem there exists $\widehat{z} \in Z$ such that $g(\widehat{z})=\widehat{z}$. So we have $\sum_{\ell \in N \backslash\{k\}} f_{k \ell}(\widehat{z})=0$ for all $k \in N$. Now, (4) and (2) of Lemma 3.5 imply that $f_{k \ell}(\widehat{x})=0$ for all $k \in N$ and $\ell \in N \backslash\{k\}$. The proof is complete by (3) of Lemma 3.5 .

q.e.d.

Remark 3.7 In addition to 2-SIVA, NE, PO, COV, RGP, and CRGP, nonsymmetric prekernels have many further properties in common with the prekernel. We mention only two of them (see Sections 7 and 9 of Orshan (1994)). (1) Any NSPK applied to any 3-person game is a singleton. (2) Any NSPK applied to any convex games is a singleton in the core of the game. The proofs are generalizations of the proofs for the prekernel due to Davis and Maschler (1965) and Maschler, Peleg, and Shapley (1972).

\section{Nonsymmetric Prenucleoli and the Positive Core}

In order to show that AN is logically independent of the remaining axioms in Sobolev's axiomatization of the prenucleolus (Theorem 2.1), Sudhölter (1993) constructed the following example of a nontrivial $\operatorname{NSPN}$. Let $(N, v)$ be a game. The positive core of $(N, v)$ is the set $\mathcal{C}_{+}(N, v)=Z(N, v, \nu(N, v))$, that is,

$$
\mathcal{C}_{+}(N, v)=\left\{x \in X^{*}(N, v) \mid(e(S, x, v))_{+}=e(S, \nu(N, v), v)_{+} \text {for all } S \subseteq N\right\} .
$$

By definition, $\mathcal{C}_{+}(N, v)$ is a compact convex nonempty polyhedral set. This set contains the prenucleolus and it coincides with the core if the core is nonempty. The prenucleolus is a subsolution of the prekernel. Hence, by Theorem 3.6 (applied to $\sigma^{\prime}=\mathcal{P} \mathcal{K}$ and to $x=\nu(N, v)$ ), the positive core of a game intersects any of its NSPKs.

Now, let $\succeq$ be any total order relation on $U$. For any game $(N, v)$ define

$$
\sigma(N, v)=\left\{x \in \mathcal{C}_{+}(N, v) \mid x \succeq_{\text {lex }} y \text { for all } y \in \mathcal{C}_{+}(N, v)\right\},
$$

where $\succeq_{\text {lex }}$ is the lexicographic order induced by $\succeq$, that is, for $N \subseteq U$ and $x, y \in \mathbb{R}^{N}, x \succeq_{\text {lex }} y$ is defined by

$$
i \in N, y_{i}>x_{i} \Rightarrow \text { there exists } j \in N \text { such that } j \succeq i \text { and } x_{i}>x_{j} .
$$

Then $\sigma$ is an NSPN that does not coincide with the prenucleolus. Note that this example is a special case of $\sigma^{\succ}$ defined (4.7). 
Used just as an auxiliary solution in the 1990', recently it turned out that the positive core is interesting in its own right. Indeed, Orshan and Sudhölter (2010) present several characterizations by simple properties thereby providing a theoretical justification of this nonempty core extension. One of the main results is the following theorem.

Theorem 4.1 Let $|U|=\infty$ and let $\sigma$ be a solution that contains the prenucleolus ${ }^{4}$. Then $\sigma$ satisfies REAS, COV, AN, RGP, and RCP, if and only if $\sigma$ coincides with one of the following solutions: (a) The prenucleolus; (b) The positive core; (c) The relative interior of the positive core.

So we may characterize the positive core as the maximal solution that contains the prenucleolus and satisfies the foregoing 5 axioms.

It should be noted that the property that the prenucleolus is a subsolution of $\sigma$ in Theorem 4.1 may be replaced by several sets of axioms. We mention only one. We call a solution $\sigma$ convex valued (CON), if $\sigma(N, v)$ is a convex set for any game $(N, v)$. Then Theorem 4.1 remains valid if "contains the prenucleolus" is replaced by "satisfies NE and CON".

In order to present examples of two classes of NSPNs some notation is useful. A configuration of $U$ is a pair $(\mathcal{U}, \succ)$ such that $\mathcal{U} \subseteq 2^{U} \backslash\{\emptyset\}, U=\bigcup\{S \mid S \in \mathcal{U}\}$, and $\succ$ is a total order on $\mathcal{U}$. Let $(\mathcal{U}, \succ)$ be a configuration. For any $N \subseteq U$ denote $\mathcal{N}^{\succ}=\{S \cap N \mid S \in \mathcal{U}\} \backslash\{N, \emptyset\}$. If $k, \ell \in U$, then $k \sim \ell$ if, for all $S \in \mathcal{U}, k \in S$ if and only if $\ell \in S$. The relation $\sim$ is an equivalence relation and the set of equivalence classes, denoted by $\mathcal{U}^{\succ}$, is a partition of $U$. Say that $(\mathcal{U}, \succ)$ is feasible if for any finite nonempty $N \subseteq U$ and any $T \in \mathcal{N}^{\succ}$ there exists a maximal element $S(T, N)$ in $\{S \in \mathcal{U} \mid S \cap N=T\}$, i.e., if $Q \in \mathcal{U}, Q \cap N=T, Q \neq S(T, N)$, then $S(T, N) \succ Q$.

Note that a configuration $(\mathcal{U}, \succ)$ is automatically feasible, if one of the following conditions is satisfied:

$$
\begin{gathered}
\text { The inverse of } \succ \text { is a well-ordering. } \\
R \in \mathcal{U}^{\succ} \Rightarrow|\{S \in \mathcal{U} \mid R \subseteq S\}|<\infty \text { or }|\{S \in \mathcal{U} \mid R \cap S=\emptyset\}|<\infty .
\end{gathered}
$$

If $(\mathcal{U}, \succ)$ is feasible and $N$ is a finite nonempty subset of $U$, then let $\succ^{N}$ be the total order on $\mathcal{N}^{\succ}$ that is induced by $\succ$, that is, for $P, Q \in \mathcal{N}^{\succ}, P \succ^{N} Q$ if and only if $S(P, N) \succ S(Q, N)$.

Now we are ready to define the NSPN $\sigma^{\succ}$ generated by the feasible allocation $(\mathcal{U}, \succ)$. Let $N$ be a finite nonempty subset of $U$ and let $S^{1}, \ldots, S^{t}$ be determined by

$$
\mathcal{N}^{\succ}=\left\{S^{1}, \ldots, S^{t}\right\} \text { and } S^{1} \succ^{N} \cdots \succ^{N} S^{t} .
$$

For any game $(N, v)$ define

$$
\sigma_{0}^{\succ}(N, v)=\left\{x \in \mathcal{C}_{+}(N, v) \mid\left(x\left(S^{1}\right), \ldots, x\left(S^{t}\right)\right) \geq_{\text {lex }}\left(y\left(S^{1}\right), \ldots, y\left(S^{t}\right)\right) \text { for all } y \in \mathcal{C}_{+}(N, v)\right\}
$$

and note that $\sigma_{0}^{\succ}(N, v)$ is recursively determined by

$$
X^{0}=\mathcal{C}_{+}(N, v) \text { and } X^{i}=\left\{x \in X^{i-1} \mid x\left(S^{i}\right) \geq y\left(S^{i}\right) \text { for all } y \in X^{i-1}\right\} \text { for all } i=1, \ldots, t,
$$

so that $\sigma_{0}^{\succ}(N, v)=X^{t}$. Hence, $\sigma_{0}^{\succ}(N, v)$ is a nonempty convex compact polyhedral set. Recall that the nucleolus of $(N, v)$ with respect to $\sigma_{0}^{\succ}(N, v)$ is denoted by $\mathcal{N}\left((N, v), \sigma_{0}^{\succ}(N, v)\right)$ and define

\footnotetext{
${ }^{4}$ i.e., $\nu(N, v) \in \sigma(N, v)$ for any game $(N, v)$
}

$$
\sigma^{\succ}(N, v)=\mathcal{N}\left((N, v), \sigma_{0}^{\succ}(N, v)\right) .
$$


Lemma $4.2 \sigma^{\succ}$ is an NSPN.

Proof: Schmeidler (1969) shows that the nucleolus of a game with respect to a nonempty compact convex set is a singleton and the proof of COV is straightforward. In order to show RGP, let $(N, v)$ be a game and $\emptyset \neq S \subseteq N$. Let $\mathcal{R}^{N}=\left\{R \cap N \mid R \in \mathcal{U}^{\succ}\right\} \backslash\{\emptyset\}$, that is, $\mathcal{R}^{N}$ is the coalition structure of $N$ generated by the partition $\mathcal{U}^{\succ}$ of $U$. Note that, for any $x \in \sigma_{0}^{\succ}(N, v)$,

$$
\sigma_{0}^{\succ}(N, v)=\left\{y \in \mathcal{C}_{0}(N, v) \mid y(R)=x(R) \text { for all } R \in \mathcal{R}^{N}\right\}
$$

Moreover, note that $\mathcal{R}^{S}=\left\{R \cap S \mid R \in \mathcal{R}^{N}\right\} \backslash\{\emptyset\}$, that is, $\mathcal{R}^{S}$ is the coalition structure of $N$ reduced to $S$. This fact together with RGP and RCP of $\mathcal{C}_{+}$implies that $\sigma_{0}^{\succ}$ satisfies RGP and RCP as well.

Let the derived game $\left(N, v_{\succ}\right)$ be the game that differs only inasmuch as $v_{\succ}(R)=x(R)$ for any $R \in \mathcal{R}^{N}$ and any $x \in \sigma_{0}^{\succ}(N, v)$. By RGP and RCP of $\sigma^{0},\left(v^{S, x}\right)_{\succ}=\left(v_{\succ}\right)^{S, x}$ for any $x \in \sigma_{0}^{\succ}(N, v)$. Now, $\widehat{x}=\sigma^{\succ}(N, v)$ coincides with the prenucleolus of the game $\left(N, v_{\succ}, \mathcal{R}^{N}\right)$ with coalition structure $\left(N, v_{\succ}, \mathcal{R}^{N}\right)$. As the prenucleolus on games with coalition structures satisfies RGP (see, e.g., Peleg and Sudhölter (2003, Theorem 5.2.7)), our proof is complete.

q.e.d.

Any NSPN satisfies 2-SIVA, NE, PO, COV, and RGP. As CRGP replaces maximality, the NSPK is a subsolution of a unique NSPK. Let $(\mathcal{U}, \succ)$ be a feasible configuration. We now determine the NSPK $\sigma$ that contains $\sigma^{\succ}$. In view of Theorem 3.4 just $\boldsymbol{a}^{\sigma}=\left(a_{k \ell}^{\sigma}\right)_{k, \ell \in U, k \neq \ell}$ has to be determined. Let $k, \ell \in U$, $k \neq \ell$, and let $P, Q \in \mathcal{U}^{\succ}$ be determined by $k \in P$ and $\ell \in Q$, and let $N=\{k, \ell\}$, let $(N, v)$ be the $0-1$ normalized game, and let $x$ be the unique element of $\sigma^{\succ}(N, v)$, i.e., $x_{k}=a_{k \ell}^{\sigma}, x_{\ell}=a_{\ell k}^{\sigma}$. The following 2 cases may occur:

(1) $P=Q$ : Then $x=\nu(N, v)$ so that $a_{k \ell}^{\sigma}=\frac{1}{2}$.

(2) $P \neq Q$ : Then $\mathcal{N}^{\succ}=\{\{k\},\{\ell\}\}$ and there are two possible subcases: If $\{k\} \succ^{N}\{\ell\}$, then $a_{k l}^{\sigma}=1$. If $\{\ell\} \succ^{N}\{k\}$, then $a_{k l}^{\sigma}=0$.

Note that the foregoing considerations just depend on the equivalence classes $P$ and $Q$ and not on their representatives $k$ and $\ell$. Hence, there exists a maximal element, denoted by $S(P)$, in $\mathcal{U}$ that contains $P$ for any $P \in \mathcal{U}^{\succ}$. By a slight abuse of notation we write $P \succ Q$ if $S(P) \succ S(Q)$. These observations show that $\sigma$ is determined as follows:

$$
\begin{aligned}
& a_{k \ell}^{\sigma}=1 \text { for all } k \in P, \ell \in Q, P, Q \in \mathcal{U}^{\succ} \text { with } P \succ Q \\
& a_{k \ell}^{\sigma}=0 \text { for all } k \in P, \ell \in Q, P, Q \in \mathcal{U}^{\succ} \text { with } Q \succ P \\
& a_{k \ell}^{\sigma}=\frac{1}{2} \text { for all } k, \ell \in P, P \in \mathcal{U}^{\succ} .
\end{aligned}
$$

Thus, $a_{k \ell}^{\sigma} \in\left\{0, \frac{1}{2}, 1\right\}$ for any $k, \ell \in U, k \neq \ell$.

Conversely, let $\sigma$ be an NSPK such that $a_{k \ell}=a_{k \ell}^{\sigma} \in\left\{0, \frac{1}{2}, 1\right\}$ for any $k, \ell \in U, k \neq \ell$. We shall present two special examples of feasible configurations that generate an NSPN in $\sigma$. For any distinct players $k, \ell \in U$ say that $k \sim \ell$ if $a_{k \ell}=\frac{1}{2}$. Let $\mathcal{U}$ denote the set of equivalence classes with respect to $\sim$ and define $\mathcal{U}_{c}=\{U \backslash R \mid R \in \mathcal{U}\}$. Moreover, define $\succ$ on $\mathcal{U}$ by $P \succ R$ iff $a_{k \ell}=1$ for any $k \in P$ and $\ell \in R$ for all $P, R \in \mathcal{U}$, and define $\succ_{c}$ of $\mathcal{U}_{c}$ by $U \backslash R \succ_{c} U \backslash P$ if $P \succ R$ for all $P, Q \in \mathcal{U}$. By Theorem 3.4 and $(4.3),(\mathcal{U}, \succ)$ and $\left(\mathcal{U}_{c}, \succ_{c}\right)$ are feasible configurations. Moreover, $\mathcal{U}^{\succ}=\left(\mathcal{U}_{c}\right)^{\succ^{c}}=\mathcal{U}$ and by the above 
construction, $\sigma^{\succ}$ and $\sigma^{\succ c}$ are subsolutions of $\sigma$. The next example shows that $\sigma^{\succ} \neq \sigma^{\succ c}$ provided that $|\mathcal{U}| \geq 4$.

Example 4.3 Let $\sigma$ be an NSPK defined by $a_{k \ell} \in\left\{0, \frac{1}{2}, 1\right\}, k, \ell \in U, k \neq \ell$. We assume that $\sigma$ generates at least 4 equivalence classes. Hence, we may assume without loss of generality that $a_{12}=a_{23}=a_{34}=1$. Let $N=\{1,2,3,4\}$ and let $(N, v)$ be defined by

$$
v(\emptyset)=v(N)=v(\{1,2\})=v(\{1,3\})=v(\{2,4\})=v(\{3,4\})=0 \text { and } v(S)=-2, \text { otherwise. }
$$

With $x=(1,-1,-1,1)$ it is straightforward to verify that $\mathcal{C}(N, v)$ is the convex hull of $x$ and $-x$. Moreover, if $S^{1}, \ldots, S^{t}$ are defined by (4.4), then $S^{i}=\{i\}$ and $t=4$ so that the set $X^{1}$ of (4.6) is defined by $X^{1}=\left\{z \in \mathcal{C}(N, v) \mid z_{1} \geq y_{1}\right.$ for all $\left.y \in \mathcal{C}(N, v)\right\}$ so that $X^{1}=\{x\}$ an, hence $\sigma^{\succ}(N, v)=\{x\}$. In the other case, if the $S^{i}$ are defined as in (4.4), but now for $\succ_{c}$ rather than $\succ$, then $S^{i}=N \backslash\{5-i\}, t=4$, and $X^{1}=\left\{z \in \mathcal{C}(N, v) \mid z(\{1,2,3\}) \geq y(\{1,2,3\}\right.$ for all $y \in \mathcal{C}(N, v)\}$. We conclude that $\sigma^{\succ_{c}}(N, v)=\{-x\}$.

The next example shows that there exist NSPKs with $a_{k \ell} \notin\left\{0, \frac{1}{2}, 1\right\}$ for some $k, \ell \in U$.

Example 4.4 Let $\sigma$ be an NSPK such that $a_{12} \notin\left\{0, \frac{1}{2}, 1\right\}$ and $a_{k \ell} \in\{0,1\}$ for all $k, \ell \in U, k \neq \ell$, with $\{k, \ell\} \neq\{1,2\}$. Let $\sigma^{\prime}$ be the NSPK that differs from $\sigma$ only inasmuch as $a_{12}=a_{21}=\frac{1}{2}$ and let $(\mathcal{U}, \succ)$ be any feasible configuration such that $\sigma^{\succ}$ is a subsolution of $\sigma^{\prime}$. The NSPN in $\sigma, \widetilde{\sigma}$, is defined as follows. Let $(N, v)$ be a game. If $\{1,2\} \nsubseteq N$, then $\widetilde{\sigma}(N, v)=\sigma^{\succ}(N, v)$. If $\{1,2\} \subseteq N$, then let $x$ be the unique element of $\sigma^{\succ}(N, v)$. If $s_{12}(x, v) \geq 0$, then define $\sigma(N, v)=\{x\}$. If $\alpha:=s_{12}(x, v)<0$, then let $y \in \mathbb{R}^{N}$ differ from $x$ only inasmuch as $y_{1}=x_{1}+\varepsilon$ and $y_{2}=x_{2}-\varepsilon$, where $\varepsilon=\alpha\left(a_{21}-a_{12}\right)$. As $s_{21}(x, v)=\alpha$, we may conclude that $a_{12} s_{21}(y, v)=a_{21} s_{12}(y, v)$ so that $y \in \sigma(N, v)$. Define $\widetilde{\sigma}(N, v)=\{y\}$. The proof that $\widetilde{\sigma}$ satisfies RGP is straightforward.

Though we do not have a complete characterization of NSPNs in general, an interesting result may be deduced under some "innocent" further condition. This condition "only" requires that infinitely many potential players have to be treated equally. As, in view of Remark $2.4,|U|=\infty$ has anyway to be assumed, this condition may be satisfied and may be regarded as not very demanding.

Theorem 4.5 Let $U^{\prime} \subseteq U$ be an infinite set. If $\sigma$ is an NSPN such that $\sigma$ satisfies $\mathrm{ETP}$ on the set of all games $(N, v)$ with $N \subseteq U^{\prime}$, then $\sigma$ is a subsolution of the positive core.

Note that under the assumptions of the foregoing theorem, if $U \backslash U^{\prime} \neq \emptyset$, then there is a nontrivial NSPN. Indeed, as shown above, there exists an NSPN in any NSPK defined by a mapping $\boldsymbol{a}=\left(a_{k \ell}\right)_{k, \ell \in U, k \neq \ell}$ that generates legal chains and satisfies $a_{k \ell}=\frac{1}{2}$ for $k, \ell \in U^{\prime}$ and $a_{k \ell} \in\left\{0, \frac{1}{2}, 1\right\}$, otherwise. If, for at least one $\ell \in U \backslash U^{\prime}, a_{k \ell}=1$ (or $\alpha_{k \ell}=0$ ) for all $k \in U^{\prime}$, then the NSPN does not coincide with the prenucleolus. However, the NSPN inherits ETP from the NSPK on the set of games $(N, v)$ with $N \subseteq U^{\prime}$, and ETP implies AN (see Footnote 2).

In order to show Theorem 4.5, the following lemma, whose proof is postponed to Section 5 , is useful.

Lemma 4.6 Let $|U|=\infty$, let $\sigma$ be an $\mathrm{NSPN}$, let $(N, v)$ be a game, let $\{x\}=\sigma(N, v)$, let $\pi: N \rightarrow U$ be an injection, and let $(\pi(N), u)$ be given by

$$
u(\pi(S))=\max \{v(S), x(S)\} \text { for all } S \subseteq N .
$$


Then $\sigma(\pi(N), u)=\{\pi x\}$.

Proof of Theorem 4.5: By Orshan's (1993) modification of Theorem 2.1, for any game $\left(N^{\prime}, v^{\prime}\right)$ with $N^{\prime} \subseteq U^{\prime}, \sigma\left(N^{\prime}, v^{\prime}\right)$ coincides with the prenucleolus. Now, let $(N, v)$ be a game and let $(\pi(N), u)$ be defined as in Lemma 4.6 such that, moreover, $\pi(N) \subseteq U^{\prime}$. With the help of a characterization of the positive core that is similar to a characterization of the prenucleolus due to Kohlberg (1971) (see (6.3.8) in Peleg and Sudhölter (2003)) it is straightforward to show that $x \in \mathcal{C}_{+}(N, v)$.

q.e.d.

Though it is not known whether any NSPN is a subsolution of the positive core, there is the following "partial" result.

Theorem 4.7 If $|U|=\infty$, if $\sigma$ is an NSPN, and if $(N, v)$ is a game such that $v(S) \geq x(S)$ for all $S \subseteq N$, where $x=\nu(N, v)$, then $\sigma(N, v)=\{\nu(N, v)\}$.

The proof of this theorem is based on the following lemma.

Lemma 4.8 Let $|U|=\infty$ and let $\sigma$ be an NSPN. Then $\sigma$ satisfies AN when restricted to the set of games that have a nonempty anticore ${ }^{5}$.

The following remark is useful.

Remark 4.9 Let $(N, v)$ be a game, $x \in \mathcal{P} \mathcal{K}_{+}(N, v)$, and $k \in N$. Then there exist $S, T \subseteq N$ such that $k \in S, k \notin T$, and $e(S, x, v)=e(T, x, v)=\max _{R \subseteq N} e(R, x, v)$. (We don't exclude the possibilities $S=N$ and $T=\emptyset$.)

Proof of Lemma 4.8: Let $(N, v)$ be a game that has a nonempty anticore, let $\pi: N \rightarrow U$ be an injection, let $N^{\prime}=\pi(N)$, let $v^{\prime}=\pi v$, and let $\sigma(N, v)=\{x\}$. It has to be shown that $\sigma\left(N^{\prime}, v^{\prime}\right)=\{\pi(x)\}$. By COV of $\sigma$ and of the anticore, we may assume that $v(N)=0$ and $v(S) \geq 0$ for all $S \subseteq N$. Let $k \in N$. By the infinity assumption on $|U|$, we may assume that $N^{\prime}=(N \backslash\{k\}) \cup\left\{k^{*}\right\}$ for some $k \in N, k^{*} \in U \backslash N$, and that $\pi(k)=k^{*}$ and $\pi(\ell)=\ell$ for all $\ell \in N \backslash\{k\}$. Let $\alpha \leq-\max _{S \subseteq N} v(S)$, let $\widetilde{N}=N \cup\left\{k^{*}\right\}$, and let the game $(\tilde{N}, w)$ be defined by

$$
w(S)= \begin{cases}v(S) & , \text { if } S \subseteq N \backslash\{k\}, \\ v\left(S \backslash\left\{k^{*}\right\}\right) & , \text { if }\left\{k, k^{*}\right\} \subseteq S, \\ \alpha & , \text { otherwise }\end{cases}
$$

for any $S \subseteq \widetilde{N}$. Let $z=\sigma(\widetilde{N}, w)$. It remains to show that, for all $S \subseteq N^{\prime}$,

$$
w^{N^{\prime}, z}(S)= \begin{cases}v(S) & , \text { if } k^{*} \notin S, \\ v\left(\left(S \backslash\left\{k^{*}\right\}\right) \cup\{k\}\right)-z_{k} & , \text { if } k^{*} \in S .\end{cases}
$$

Indeed, if (4.13) is valid, then, by COV and RGP, $\{\pi(x)\}=\sigma\left(N^{\prime}, v^{\prime}\right)$.

Assume, on the contrary, that (4.13) is not valid. Then two cases may occur:

\footnotetext{
${ }^{5}$ The anticore of a game $(N, v)$ is the set of all $x \in X(N, v)$ such that $x(S) \leq v(S)$ for all $S \subseteq N$.
} 
(1) There exists $S \subseteq N \backslash\{k\}$ such that $v(S)=w(S)<w(S \cup\{k\})-z_{k}=\alpha-z_{k}$. As $v(S) \geq 0$, $w(\{k\})-z_{k}=\alpha-z_{k}>0$. We conclude that $s_{k k^{*}}(z, w)>0$. As $z \in \mathcal{P} \mathcal{K}_{+}(\tilde{N}, w), s_{k^{*} k}(z, w)=$ $s_{k k^{*}}(z, w)$. As $k$ and $k^{*}$ are substitutes, $z_{k^{*}}=z_{k}$. Now, let $T \subseteq \widetilde{N}$ such that $k \notin T$. If $k^{*} \notin T$, then

$$
e(T, z, w)=v(T)-z(T)<v(T \cup\{k\})-z(T)-z_{k}-z_{k^{*}}=e\left(T \cup\left\{k, k^{*}\right\}, z, w\right),
$$

because $-z_{k}-z_{k^{*}}=-2 z_{k}>-2 \alpha \geq \max _{S, T \subseteq N} v(S)-v(T)$. If $k^{*} \in T$, then

$$
e(T, z, w)=\alpha-z(T)<v\left(\left(T \backslash\left\{k^{*}\right\}\right) \cup\{k\}\right)-z(T)-z_{k}=e(T \cup\{k\}, z) .
$$

In view of Remark 4.9 the desired contradiction has been obtained.

(2) There exists $S \subseteq N \backslash\{k\}$ such that $v(S \cup\{k\})-z_{k}=w\left(S \cup\left\{k, k^{*}\right\}\right)-z_{k}<w(S \cup\{k\})=\alpha$. As $v(S \cup\{k\}) \geq 0, z_{k}>-\alpha$ so that $z(\widetilde{N} \backslash\{k\})=-z_{k}<\alpha$. We conclude that $s_{k^{*} k}(w, z) \geq$ $e(\tilde{N} \backslash\{k\}, z, w)=\alpha+z_{k}>0$ and, as in the first case, $z_{k}=z_{k^{*}}$, because $k$ and $k^{*}$ are substitutes. Let $S \subseteq \widetilde{N}$ with $k \in S$. If $k^{*} \in S$, then

$$
e(S, z, w)=v\left(S \backslash\left\{{ }^{*}\right\}\right)-z(S)<v\left(S \backslash\left\{k, k^{*}\right\}\right)-z\left(S \backslash\left\{k, k^{*}\right\}\right)=e\left(S \backslash\left\{k, k^{*}\right\}, z, w\right),
$$

because $z_{k}+z_{k^{*}}>-2 \alpha \geq \max _{S, T \subseteq N} v(S)-v(T)$. If $k^{*} \notin S$, then $e(T, z, w)=\alpha-z(T)<$ $e(T \backslash\{k\}, z, w)$ and, hence, the Remark 4.9 again yields the desired contradiction.

q.e.d.

Proof of Theorem 4.7: By COV, we may assume that $\nu(N, v)=0 \in \mathbb{R}^{N}$. According to Sobolev (1975), there exists a transitive game ${ }^{6}$ game $(M, w)$ that satisfies the following properties:

(1) $N \subseteq M$.

(2) $w(T) \in\{v(S) \mid S \subseteq N\}$ for all $T \subseteq M$ and $w(M)=v(N)=0$.

(3) With $z=0 \in \mathbb{R}^{M}, w^{N, z}=v$.

By (2), $w(S) \geq 0$ for all $S \subseteq M$ and $w(M)=0$ so that $z$ is in the anticore of $(M, w)$. By Lemma 4.8 and PO, $\{z\}=\sigma(M, w)$. By RGP and $(3),\{\nu(N, v)\}=\sigma(N, v)$.

q.e.d.

Remark 4.10 Let $\sigma$ be an NSPN. If $\sigma(N, v)=\{\nu(N, v)\}$ for any game $(N, v)$ that has a nonempty anticore, then, by Lemma 4.6 - indeed, the game $(\pi(N), u)$ has a nonempty anticore $-\sigma$ is a subsolution of the positive core. Note however, that the foregoing proof cannot be used to show that $\{\nu(N, v)\}=\sigma(N, v)$ for any game that has a nonempty anticore, because the game $(M, w)$ may not inherit this property of having a nonempty anticore.

\section{The Proof of Lemma 4.6}

As $|U|=\infty$, we may assume that $\pi(N) \cap N=\emptyset$. Denote $\pi(i)=i^{*}$ for all $i \in N, S^{*}=\left\{i^{*} \mid i \in S\right\}$ for every $S \subseteq N$, and $M=N \cup N^{*}$. Let $\beta=\max _{S, T \subseteq N} v(S)-v(T)$ and $\alpha<-2|N| \beta$. Let $(M, w)$ be defined

\footnotetext{
${ }^{6} \mathrm{~A}$ game is transitive if its symmetry group is transitive.
} 
be the following formula, where $S, T \subseteq N$ :

$$
w\left(S \cup T^{*}\right)= \begin{cases}v(S) & , \quad \text { if } S=T \\ 0 & , \quad \text { if } S=\emptyset \\ v(N) & , \quad \text { if } S=N \text { and } T=\emptyset \\ \alpha & , \text { otherwise }\end{cases}
$$

Let $\{y\}=\sigma(M, w), \mu=\max _{S, T \subseteq N} e\left(S \cup T^{*}, y, w\right)$, and $\rho=\max _{k \in N}\left(s_{k k^{*}}(y, w)\right)_{+}$. Note that $\rho=$ $\max _{k \in N}\left(s_{k^{*} k}(y, w)\right)_{+}$by Corollary 3.3 .

If suffices to show that $\rho=0$. Indeed, if $\rho=0$, then $y(N) \geq v(N)$ and $y_{N^{*}} \geq 0$. Hence, by Pareto optimality of $y, y(N)=v(N)$ and $y_{N^{*}}=0$, thus $w^{N, y}=v$ by (5.14) and $y_{N}=x$ by RGP. Moreover, by REAS, $x_{i} \geq-\beta$ for all $i \in N$, thus $w^{N^{*}, y}\left(S^{*}\right)=(v(S)-x(S))_{+}$for every $S \subseteq N$. Hence, COV completes the proof.

In order to show that $\rho=0$ we assume, on the contrary, $\rho>0$ and proceed by showing the following 7 claims which finally leads to the desired contradiction. Let

$$
N_{0}=\left\{i \in N \mid y_{i}<0\right\}, \overline{N_{0}}=\left\{i \in N \mid y_{i} \leq 0\right\}, N_{0}^{\prime}=\left\{i \in N \mid y_{i^{*}}<0\right\} \text {, and } \overline{N_{0}^{\prime}}=\left\{i \in N \mid y_{i^{*}} \leq 0\right\} .
$$

Claim 1: $e(N, y, w)<\mu$ : Assume the contrary. Then $s_{k^{*} k}(y, w)=s_{k k^{*}}(y, w)=\mu>0$ for all $k \in N$ by Corollary 3.3. By Pareto optimality of $y$ there exists $i \in N$ with $y_{i^{*}}>0$. Let $s_{i^{*} i}(y, w)(=\mu)$ be attained by $S \cup T^{*}$, that is,

$$
S, T \subseteq N, i \notin S, i \in T \text {, and } e\left(S \cup T^{*}, y, w\right)=\mu .
$$

By $(5.14), w\left(S \cup T^{*}\right) \leq w\left(S \cup\left(T^{*} \backslash\left\{i^{*}\right\}\right)\right)$, thus

$$
\mu=e\left(S \cup T^{*}, y, w\right) \leq w\left(S \cup\left(T^{*} \backslash\left\{i^{*}\right\}\right)\right)-y(S)-y\left(T^{*}\right)<e\left(S \cup\left(T^{*} \backslash\left\{i^{*}\right\}\right), y, w\right),
$$

which is impossible.

Claim 2: $\rho<\mu$ : Assume the contrary and let $k \in N$ satisfy $s_{k k^{*}}(y, w)=\mu$. Let $s_{k k^{*}}(y, w)$ be attained by $S \cup T^{*}$. By Claim $1, w\left(S \cup T^{*}\right)=\alpha$. For every pair $\left(Q, Q^{\prime}\right)$ satisfying $N_{0} \subseteq Q \subseteq \overline{N_{0}}$ and $N_{0}^{\prime} \subseteq Q^{\prime} \subseteq \overline{N_{0}^{\prime}}$ we have

$$
\mu \geq e\left(Q \cup Q^{\prime *}, y, w\right) \geq \alpha-y(Q)-y\left(Q^{\prime *}\right) \geq \alpha-y(S)-y\left(T^{*}\right)=\mu,
$$

thus all inequalities of (5.15) are, in fact, equalities. Hence, $N_{0} \subseteq S \subseteq \overline{N_{0}}, N_{0}^{\prime} \subseteq T \subseteq \overline{N_{0}^{\prime}}$, and

$$
\left(N_{0} \backslash \overline{N_{0}^{\prime}}\right) \cup\left(N_{0}^{\prime} \backslash \overline{N_{0}}\right) \neq \emptyset .
$$

Indeed, (5.16) follows from the inequality $\alpha-y(R)-y\left(R^{*}\right)<v(R)-y(R)-y\left(R^{*}\right)$ which is true for every $R \subseteq N$.

Two cases may occur:

(1) $N_{0} \backslash \overline{N_{0}^{\prime}} \neq \emptyset$ : Then there exists $i \in N_{0} \backslash \widetilde{N}_{0}$. If additionally $\left|N_{0}\right| \geq 2$, then

$$
s_{i i^{*}}(y, w) \geq e\left(N_{0} \cup N_{0}^{\prime *}, y, w\right)=\alpha-y\left(N_{0}\right)-y\left(N_{0}^{\prime *}\right)=\mu>e\left(\left(N_{0} \backslash\{i\}\right) \cup N_{0}^{\prime *} \cup\left\{i^{*}\right\}, y, w\right) .
$$

Thus $s_{i^{*} i}(y, w)$ is attained by $N_{0}^{\prime *} \cup\left\{i^{*}\right\}$ in any case. As $y_{i^{*}}>0, e\left(N_{0}^{\prime *} \cup\left\{i^{*}\right\}, y, w\right)<e\left(N_{0}^{\prime *}, y, w\right)$. Hence $N_{0}^{\prime} \neq \emptyset$ and $s_{\ell^{*} \ell}(y, w)>\mu$ for all $\ell \in N_{0}^{\prime}$ which is impossible. 
(2) $N_{0}^{\prime} \backslash \overline{N_{0}} \neq \emptyset$ : Then there exists $j \in N_{0}^{\prime} \backslash \overline{N_{0}}$. By Claim 1 ,

$s_{j^{*} j}(y, w)=e\left(N_{0} \cup{N_{0}^{\prime *}}^{*}, y, w\right)=\mu>\max \left\{e\left(N_{0} \cup\{j\} \cup\left(N_{0}^{\prime *} \backslash\left\{j^{*}\right\}\right), y, w\right), e(N, y, w)\right\}=s_{j j^{*}}(y, w)$ so that the desired contradiction has been obtained.

Claim 3: $y_{k}+y_{k^{*}} \geq-\beta$ for all $k \in N$ : Assume, on the contrary, that there exists $k \in N$ with $y\left(\left\{k, k^{*}\right\}\right)<-\beta$. By Remark 4.9 there exists $S \cup T^{*} \subseteq M$ with $e\left(S \cup T^{*}, y, w\right)=\mu$ and $k \notin S$. By Claim $2, k \notin T$. However,

$$
w\left(S \cup\{k\} \cup T^{*} \cup\left\{k^{*}\right\}\right) \geq w\left(S \cup T^{*}\right)-\beta,
$$

thus $e\left(S \cup\{k\} \cup T^{*} \cup\left\{k^{*}\right\}, y, w\right)>e\left(S \cup T^{*}, y, w\right)=\mu$ which is impossible.

Claim 4: $e(N, y, w)<\rho:$ Assume the contrary. As in the proof of Claim 1, by PO, there exists $i \in N$ with $y_{i^{*}}>0$. Let $s_{i^{*} i}(y, w)\left(=s_{i^{*} i}(y, w)=\rho\right)$ be attained by $S \cup T^{*}$. Then

$$
e\left(S \cup\left(T^{*} \backslash\left\{i^{*}\right\}\right), y, w\right)>e\left(S \cup T^{*}, y, w\right)=\rho>0
$$

implies $S=T \backslash\{i\} \neq \emptyset$. As $v\left(S \cup T^{*}\right)=\alpha,(5.17)$ implies

$$
\alpha>y(S)+y\left(T^{*}\right)>y(S)+y\left(S^{*}\right) .
$$

Now, (5.18) is impossible by Claim 3 .

Claim 5: $\rho \leq \alpha-y\left(N_{0}\right)-y\left(N_{0}^{\prime *}\right):$ Let $k \in N$ satisfy $s_{k k^{*}}(y, w)=\rho$. By Claim $4 s_{k k^{*}}(y, w)$ is attained by $S \cup T^{*} \subseteq M$ satisfying $w\left(S \cup T^{*}\right)=\alpha$. Hence our claim follows immediately.

Claim 6: $N_{0}^{\prime} \subseteq \overline{N_{0}}$ : Assume, on the contrary, there exists $k \in N_{0}^{\prime} \backslash \overline{N_{0}}$. By Claim 5 ,

$$
s_{k^{*} k}(y, w) \geq e\left(N_{0} \cup N_{0}^{\prime *}, y, w\right) \geq \alpha-y\left(N_{0}\right)-y\left(N_{0}^{\prime *}\right) \geq \rho,
$$

thus $s_{k^{*} k}(y, w)=\rho$. By Claim 4 ,

$$
s_{k k^{*}}(y, w)=e\left(N_{0} \cup\{k\} \cup\left(N_{0}^{\prime *} \backslash\left\{k^{*}\right\}\right), y, w\right)<\alpha-y\left(N_{0}\right)-y\left(N_{0}^{\prime *}\right),
$$

which is impossible.

Claim 7: $N_{0} \subseteq \overline{N_{0}^{\prime}}$ : Assume, on the contrary, there exists $k \in N_{0} \backslash \overline{N_{0}^{\prime}}$. By Claim 5,

$$
s_{k k^{*}}(y, w) \geq e\left(N_{0} \cup N_{0}^{\prime *}, y, w\right) \geq \alpha-y\left(N_{0}\right)-y\left(N_{0}^{\prime *}\right) \geq \rho,
$$

thus $s_{k k^{*}}(y, w)=\rho$. As $e\left(\left(N_{0} \backslash\{k\}\right) \cup N_{0}^{\prime *} \cup\left\{k^{*}\right\}, y, w\right)<\alpha-y\left(N_{0}\right)-y\left(N_{0}^{\prime *}\right), s_{k^{*} k}(y, w)$ must be attained by $N_{0}^{\prime *} \cup\left\{k^{*}\right\}$. Hence, $N_{0}^{\prime} \neq \emptyset$. The observation

$$
\rho \geq e\left(N_{0}^{\prime *}, y, w\right)>-y\left(N_{0}^{\prime *}\right)-y_{k^{*}}=e\left(N_{0}^{\prime *} \cup\left\{k^{*}\right\}, y, w\right)
$$

yields a contradiction.

Now the proof can be finished. By Claims 6 and 7

$$
y\left(N_{0} \cup N_{0}^{\prime}\right)=y\left(N_{0}\right) \text { and } y\left(N_{0}^{*} \cup N_{0}^{\prime *}\right)=y\left(N_{0}^{\prime *}\right)
$$

and, by Claim 5, $y\left(N_{0}\right)+y\left(N_{0}^{\prime *}\right)<\alpha-\rho<\alpha$. On the other hand, by Claim 3 ,

$$
y\left(N_{0} \cup N_{0}^{\prime}\right)+y\left(N_{0}^{*} \cup N_{0}^{\prime *}\right) \geq-|N| \beta>\alpha,
$$

which is impossible.

q.e.d. 


\section{References}

Aumann, R. J., And M. Maschler (1985): "Game theoretic analysis of a bankruptcy problem from the Talmud", Journal of Economic Theory, 36, 195 - 213.

Davis, M., And M. Maschler (1965): "The kernel of a cooperative game", Naval Research Logistics Quarterly, 12, 223-259.

(1967): "Existence of stable payoff configurations for cooperative games", in Essays in Mathematical Economics in Honor of Oskar Morgenstern, ed. by M. Shubik, pp. 39 - 52, Princeton, N.J. Princeton University Press.

Hwang, Y.-A., And P. Sudhölter (2001): "Axiomatizations of the core on the universal domain and other natural domains", International Journal of Game Theory, 29, 597 - 623.

KohlBerg, E. (1971): "On the nucleolus of a characteristic function game", SIAM Journal on Applied Mathematics, 20, $62-66$.

Maschler, M., B. Peleg, and L. S. Shapley (1972): "The kernel and bargaining set for convex games", International Journal of Game Theory, 1, 73 - 93.

Orshan, G. (1993): "The prenucleolus and the reduced game property: Equal treatment replaces anonymity", International Journal of Game Theory, 22, $241-248$.

— (1994): "Non-symmetric prekernels", Discussion paper 60, Center for Rationality, The Hebrew University of Jerusalem.

Orshan, G., And P. Sudhölter (2003): "Reconfirming the prenucleolus", Mathematics of Operations Research, 28, $283-293$.

(2010): "The positive core of a cooperative game", International Journal of Game Theory, 39, $113-136$.

PeleG, B. (1986): "On the reduced game property and its converse", International Journal of Game Theory, 15, $187-200$.

Peleg, B., And P. Sudhölter (2003): Introduction to the Theory of Cooperative Games. Kluwer Academic Publishers, Dordrecht.

SchmeIDLER, D. (1969): "The nucleolus of a characteristic function game", SIAM Journal on Applied Mathematics, 17, 1163 - 1170.

Sobolev, A. I. (1975): "The characterization of optimality principles in cooperative games by functional equations", in Mathematical Methods in the Social Sciences, ed. by N. N. Vorobiev, Vol. 6, pp. 95 151, Vilnius. Academy of Sciences of the Lithuanian SSR, in Russian.

SudhöLter, P. (1993): "Independence for characterizing axioms of the pre-nucleolus", Working paper 220, Institute of Mathematical Economics, University of Bielefeld.

Sudhölter, P., And B. Peleg (2000): "The positive prekernel of a cooperative game", International Game Theory Review, 2, 287 - 305. 